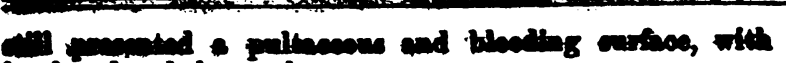

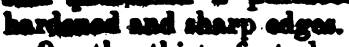

- in the thirts-fint day, the ourfice of the ulcer was bening cleener and its edres lese prominent: the codems and exlling had disppeared, but the edges of the wound left by the suppurated gland were ulcerated, and had a sFphilitic appearance.

On the forty-finst day, this sore had again assumed a phagedenic surface. Its edges were undermincd, inflamed, and very painful. The pus discharged was tinged with blood. The wound resulting from the open bubo was becoming clean, but its edges were undermined and sharp.

$\Delta$ fortnight after this, the surface of the sore again became hollthy; and ten days later, the bubo began to cicatrise.

After lasting from the 21st of August to the 29th of Ootober, the sore was all but healed, and the bubo resulting from it was completely cicatrised.

M. Robert had at this time regained his habitual health, and subsequently experienced no farther inconvenience. The cats also remained well and showed no indications of having had their constitutions affected, nor did they suffer from enlargement of the glands consequent upon the inoculations.

From his experiments, M. Robert arrives at the conclusion that-

The syphilitic virus inoculated upon the ears of cats determines a slight ulceration which exists for eight or ten days and secretes a purulent fluid.

When this pus is inoculated on the same or on another animal it occasions an ulceration exactly resembling the one from which it was taken. When inoculated on man it gives rise io a chancre in the same way as if the syphilitic poison had been taken directly from man.

The ulceration in animals lasts on an arerage from eight to ten days.

It occesions in them neither inflammation of the absorbent nor secondary symptoms.

When several inoculations are made on the same animal, whether the pus be taken from the animal itself or from man, the last are neither more or less intense than the first. They all follow exactly the same course.

In this last respect, M. Robert's experience is at variance with that of $M$. Auzias Turenne.

\section{NOTES ON EPIDEMICAL DISEASES.}

\section{By WILLLAM ADDISON, M.D., F.R.S.}

$$
\text { No. IV. }
$$

INFLUENZA, CHOLERA, CONTINUED FEVER, TYPHUS, AGUE, REXITTENT FEVER, DIARRHEA, DYBENTERY, SKALL-POX, GCARLET FEVER, YEASLES, HOOPING-COUGH.

$$
\text { IV. PERsonal catges. }
$$

Doring the prevalence of an epidemic, it is not all persons of the same age, nor all those who inhabit the same locality, that are affected-the external miasm, from whaterer source derived, must concur with some personal condition, or the malady does not make its seizure. If the public health be watched, either by medical men in the practice of large dispensaries, or by other persons, it will be found in the mortuary returns, that epidemics usually manifest themselves amongst the population in a certain fixed order : they are first noted in infants; then in children, according to their age and strength; then in the weakly or feeble; afterwards, in females and the aged; and, last of all, in the robust in the middle period of life.

It also happens that children of the same age, previously to all appearance in good health, become affected by epidemic disorders, enter upon fever, measles, scarlatina, hooping-cough, otc., under the same outward circumstances, in the same house, nay, even in the same room Yot how different the symptoms, stages, duration, and results of the malady; how various the complications and sequels! Bome are opeedily cured, other take a longer

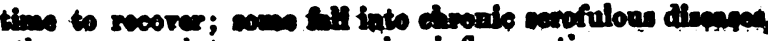
others sucoumb to a expervening inflamanation.

Thees phenomens are referred to difiereaces of conetitetion. Thus persons who show but little power in resisting sickness, or in whom disorders run an irregular couree, are spoken of as constitutionally feeble, delicate, or susceptible; and the reverse of these are the robust and strong. But why is one person of a strong, and another at the same age of a weak constitution? The answer to this question is of general interest ; it forms part of the subject of these notes ; and as it touches upon the history of organisation, I shall briefly refer to some points connected with it.

Every part of the human body, from the first moments of intra-uterine life to puberty, passes through progressive phases of growth. The embryo, footus, infant, child, and adult, experience changes of organisation; and, in proportion as each organ becomes more perfect, functions undergo corresponding changes.

Organisation, functions, and diseases, are necessarily connected with each other. New spheres of organisation develope new functions, and new functions are inevitably associated with new symptoms of disease. That is to say, until organisation and functions are completed, growth is liable to disturbances, and new functions entail a possibility of new deviations-new susceptibilities to disease.

If at any period of intra-uterine life there should occur check or departure from the normal course of growth, such becomes stamped on the body, and a source either of disease or imperfection arises. Great departures produce monstrosities; defects of a lesser kind circumscribe functions; and the inquiry is, whether the least-those compatible with the appearances of health-may not be causes of delicacy of constitution.

Passing over monstrous growths, many of which cannot live an hour after birth, we find infants born with obvious defects :-without fingers or toes; with club-feet; or with only one hand; with hare-lip; cleft-palate; a divided uvula ; or with cataract of one or both eyes; with transposition of the heart ; with only one kidney ; with hernia ; and with fractures or luxations of the limbs. These, with other irregularities of similar extent, constitute, as it were, a middle group of a series of defects. They are less than those to which we apply the term "monstrosities"; but they are greater than many minor anomalies, such, for example, as softening, or congestion of the mucous membrane of the stomach and bowels, limited capacity of chest, tubercles in the lungs, imperfect heart, small-brain, water in the head, and others which, making no outward appearance, escape detection until the death of the individual, which often happens from other circumstances, with which these anomalies have nothing to do. Moreover, a number of facts attent that the fotus may contract maladies from the parent, and that infants may be born sick, convalescent, or recovered from disease.

At birth, organisation and functions are not completed. The outline of the bones is traced, but the hard material has yet to grow. A division in the heart has to be completed. Some months elapse befure the first teeth appear. Then there is the second set to follow. After these, the epoch of puberty has to be accomplished. Moreover, several organs have at birth to begin their uffice, and therefore at that time first to show their capacity to fulfil it. The lungs, for example, are now tirst intlated with air. Upon the first inspirations, the blood experiences new changes of composition, and enters upon new courses; and new courses bring with them new liabilities to deviation. Lastly, soon after birth, the stomach and intestines have for the first time to receive and digest food. If at birth some small lobules of the lungs do not receive the air, illness arises, which may be surmounted by the gradual penetration of air into the unexpanded texture. Should this not take place, the texture becomes permanently hard and dense; the child, notwithstanding, may slowly regain the appearance of health, but it does so with anatomical changes in the structure of the lung. And it has been recorded of children who have thus been ill at birth with 
cinim of the leme thes the prow up with foable Ciritition, and liable to tedions divordens of the cheat. Anin, the air that firet enters the lung may be pure or sinere; it can scarcely fail to have the latter quality in an at romic season, and in a close, dirty, ill-rentilatod room. If if the first air which the infant inspires be charged ith noxious effluvis from any quarter, the blood is detoFiorated; and with imporerished blood circulating, if illness do not appear, assuredly the constitution cannot long romin robust. If the growth of the hard material of the bones be delayed, the child is riokety; and from this circumstance the constitution, it is well known, is said to be delicate or scrofulous. There are, then, minor deviations of organisation which are compatible with the usual appearances of heslth, but which, impairing the vigour of the constitution, are worthy the attention of the physician.

"We should remember," says M. Billard, "that the different organs in their progress of development are susceptible of acquiring certain modifications which dispose them to idiosyncrasies, the influence of which may remain during life. How often do we see children, born with all the appearances of flourishing health, become feeble and sickly from diseases which, attacking them in early infancy, leave afterwards organic changes, which time can scarcely remove, and which in some individuals never disappear! It is perhaps to pneumonia, developed during intra-uterine life or soon after birth, that short breathing, husky voices, asthmas or idiopathic coughs, with which some individuals are through life affected, are to be attributed."

If we further inquire, how under present social habits new-born infants in general encounter the new stimuli, air, food, heat, and cold? The answer is found in the tables of mortality: atelectasis, pnenmonia, diarrhoea, convulsions, teathing, etc., sweep off twenty out of every hundred before the expiration of a year. Of the remainder, one-half at least experience epidemic sickness in some shape or another, and many of these recover with the standard of health lowered, liable to various forms of scrofulous disease.

In the year 1838, the total registered deaths in England were 342,529 , and the mortality, chiefly of young children, under the heads teething, convulsions, pneumonia, diarrhos, hydrocephalus, measles, hooping-cough, and scarlet fever, was as follows:-

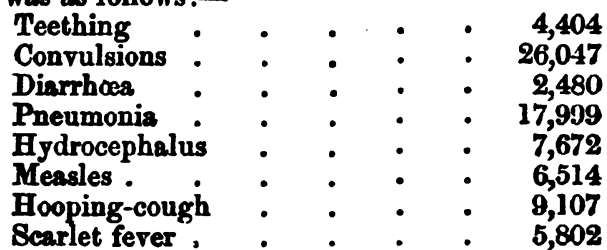

The sum of these numbers is 80,025 ; and if, for the sake of argument, we reckon for the series one death in six cases of illness, then a mortality of 80,000 will represent a sickness of 480,000 , - that is to say, for 80,000 deaths, there would be 400,000 cures or recoveries.

Now, in a record of infantile mortality from teething, convulsions, diarrhoea, and pneumonia, - or of youths dying of measles, hooping-cough, and scarlet fever, the inadequacy of the terms used must be borne in mind. They convey no information upon the extent or ramifications of the disease which has occasioned death; whereas pathological anatomy daily demonstrates in the maladies to which these terms are applied, that though one part of the body may suffer more prominently of severely than the rest, and therefore furnish the chief or characteristic symptoms; jet that many secondary and influential anatomical changes contribute to the illness, and that these sometimes usurp the foremost rank, and themselves extinguish life. Thus children who have been burned or scalded, suffer not only from the injury itself, but from supervening changes in the brain, lungs, or bowels; and when death takes place, it is alwass from the supervening malady. In acute rheumativen, the patient may be really recovering from inflammation seated in the joints, and yet death be approseching frem secondary anatomical changes epringing up in the

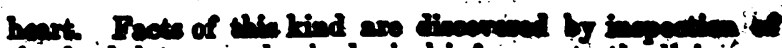
the deid, lot wo spply the logieal infarimes to the livity

If wo bok upon cearlet fover with onr minds freed thin the prejudices of authority, what do wo 20 i The becl. covered externally from head to foot with rednees, and in ternslly, as far as the eje can reach, wo 800 the redincen. continued; we have at the same time, and constituting the disease, both the external and internal surfaces of tho body inflamed, besides disorder and changes in other organs. If we scrutinize any other epidemic disease, in the same waj, Te have abundant evidence to the same effect. So that it may be stated generally, there is no severe disease unaccompanied with sundry and important collateral disturbances; and that what are termed complications, superventione and eeguels, are the more prominent of these disturbances pressing onward, evolving signs, and running their course concurrently with the chief malady, or gaining strength and importance upon its decline.

Cass. A woman, aged 37, a schoolmistress, teaching, doing for herself, and following the occupations of health, is of weak constitution, and occasionally subject to cough and pain in the side. She has at various times for Jears been for short periods under medical care, her lungs are known to be delicate. The following symptoms have been gathered at different times, and are here noted in her own words :- "She feels strings breaking at the heart; the chest feels full ; burning heats flash out all over the body: creepings under the skin; she feels just as if pins and toedles were nittling and shooting about; cold chills and pain in the chest; she feels wind in the head and back; the pit of the stomach feels as if she had lost something; stitches all over the bowels, and the pains when they are not in the bowels shoot up and dart through the chest; a dreadful beating in the head; cold shivers down the back bone, and sometimes a dashing at the chest." At length, the diseases to which these symptoms must be referred, proved fatal, and the body was examined. The left lung was found firmly adherent to the wall of the chest, and there was a cavity surrounded by tuberculous matter in it interior. In the right lung several groups of tubercles were disseminated. The liver was pale and fatty. The small intestines were covered with flakes of false membrane, and miliary tubercles were scattered upon the abdominal peritoneum.

When, therefore, it is pronounced that infants have recovered from teething, convulsions, inflammations of the lungs, or diarrhces, or that youths have recorered from measles, scarlet-fever, or hooping-cough, it must be borne in mind that the word recovery implies only a relief of the person from the symptoms of the malady, and a return of the feelings and appearances of health. It cannot mean that all the organs which have been implicated, or have suffered from the malady, have returned to their first and untouched condition; for in the living body, however much there may be in the subsidence of symptoms to lead to such a presumption, this cannot be known positively -as a fact. Anatomical changes of a secondary nature, as regards the principal disease, and which are consequences of it, may, with the recovery, gradually return to a normal state, and the whole body regain its pristine vigour, but no one can be sure of it; on the contrary, any remaining delicacy of constitution, any lingering debility, and especially the commencensent-as is too often the case- of ome scrofulous sequel, after epidemical disease, may bo confidently appealed to in proof that the reverse of this is actually the case.

But, further, what is the import of the word CURE as respects the recovery of the patient? Upon this subject it is almost needless to premise that, however much the farourable termination of an accident, or disense, mas be forwarded by medical or surgical assistance, cure is entirely the result of a power or property inherent in the animal economy, without which neither cture, repair, convalescenos, nor recovery could take place. This power' or property operates by fixed laws; and there are numerous facts proving that the cure of internal inflammation has the 
mone nagew, and the ame course, as the cure of infiammation, or ropair of sccidents, in enalogous textures outwardly. Such being the case, the cure of inflammation boing a natural process subject to fixed laws, our inquiry is at to the infwence of cure upon recovery, -i.e.: upon the constitution of the person for the future.

The cure of confluent small-pox leares pits and scars upon the skin. The eyelashes are not reproduced, and scars upon the eyelids sometimes prevent their closing. From this the eyes become weak and irritable, and the sight of an eje has been lost through the irritation occasioned by the puckering of scars produced by the healing of the pocks. Here the recovery or cure of a disease leaves the person in a worse condition than before he had the disorder; the cure impairs the function of one part of the body, and that im. pairment leads to disturbance, and it may be the destruction of another part. It is the law in the cure of a severe burn, or other accident which destroys the integument, that the true texture of the skin is not reproduced. And the cicatrix which cures the wound often stiffens a joint. A scrofulous disease may be cured, but it is so with permanent puckering in the lung, deformity of a limb, or opacity in the cornea.

The cure of pleurisy very often takes place, but very seldom without adhesion of the lung to the side. The affection of the heart, which supervenes on acute rheumatism, may be cured, in the natural history sense of the word, but it is with adhesion of the heart to the pericardium. In these cases, the cure may be complete, but the recovery is not. Where there are adhesions, limiting the function of a lung, or uniting the pericardium to the heart, the person may enjoy a species of health, but he is clearly of a delicate constitution; and in future disorders it is well if the medical attendant know of the previous condition. Those who have suffered from a sprained ankle, or broken leg, are often reminded of the occurrence. Long after the cure, upon fatigue, changes of weather, or in an illness, the enfeebled limb will be painful, and liable to swell, when the other remains unaffected.

A careful observer says that of 919 children dying of various diseases, from five to fifteen years of age, he found, on examination, that 515 , or three-fifths of them presented changes and defects in some one or more of the organs of the body, and these not the result of the particular malady which caused death, but of some former illness. Again, it is stated by an eminent physiologist, with respect to older persons whose internal condition he bad examined after death, that four-fifths presented evident incontestable traces, not of recent, but of former maladies.

Hence there is abundant evidence of sundry kinds of deformity of growth and function before and after birth; also of anatomical changes of structure, the natural result of the cure of disease, which do not appear, escaping detection except in as far as their presence may be denoted by varieties of constitution; by a low standard of health; and by tedious irregularities in the expected course of familiar disorders. $\mathbf{A}$ robust constitution is the effect of a right performance of many functions. Functions cannot be rightly performed with defects of structure or impoverished blood. And abnormal conditions of blood, as long as they continue, are equivalent to defects of structure.

The sum of the facts which have been stated proves that infants may be born with, or may acquire from early illness unsoundness of organisation, and yet maintain the appearance of health ; that children may recover from epidemical diseases with still existing collateral disturbances and weakened functions, which, though compatible with a certain ghare of health, nevertheless give complexion and irregularity to future disorders; and that older persons may resume their employments, and perhaps overlook an illness which, by circumscribing the function of an important organ, has enfeebled the constitution. If these conclusions be established, aud if it be true that infants before birth acquire tho maladies of the parent, then a sufficient reason has been established for their being born with differences of constitution.
In many instances, we are able to refor feebleness of bealth to anatomical changes of structure discorerable during life. In others, the fact is better explained by inspection after death, revealing what could not othervise have been known. Conversely, persons of delicate constitution are inferred to be subjects either to some defect of tion are inferred to be subjects remaining from a former malady, though whilst the person be living we may be unable to assert unequirocally where it is to be found. This is a sound practical conclusion, inasmuch as we are naturally led by it-whenever maladies are prolonged, or assume the irregular scrofulous typo-to a careful inquiry into the course and cure of former disorders, and a stricter examination of the person with reference to them.

And now to return to the opening of this division of our subject: the influence of personal causes upon the course and cure of epidemical diseases.

There is no difficulty in comprehending the reason why, amongst children of the same age, in the same street, house, or room, and in the same condition of life, some are ill, but recover; others recover from the chief malady, but succumb to a scrofulous sequel ; some escape illness altogether, and others die of the acute disorder. It is because there is at all times, and in all places, especially in cities and towns, a constitutionally predisposed class-individuals at all ages, pursuing the amusements, occupations, and labours of health with unsoundness of organisation, and therefore with those personal conditions which dispose to the attack of epidemic sickness.

In addition to the predispositions here indicated, and which arise from permanent anatomical change in one or more organs of the body, it is important to bear in mind, during the prevalence of an epidemic atmosphere-that is, at a time when the whole population is habitually breathing an unwholesome medium disposing to sickness, yet too much diluted to produce it unaided - that a slight or transient additional morbific influence is sufficient to cause active disease. Under such an influence, the first spark of feverishness, which casual fatigue, broken rest, want, inebriation, or the more common accidents of life may elicit, will induce the dominant malady.

Maidstone, Feb. 1854.

\section{CONDENSATION OF THE APEX OF THE LUNG, WITH DILA'TED BRONCHI.}

By JOSEPH BULLAR, M.D., Physician to the Royal South Hants Infirmary.

THE following case is important, as illustrating the morbid anatomy of one form of pulmonary disease, which must at the time hare presented many of the signs of commencing phthisis, and as showing the difficulty of diagnosis subsequently, owing to this change of structure.

Case. The patient was a young man, aged 20, who was admitted into the South Hants Infirmary, with severe compound fracture of the left thigh, fivin an injury with steam machinery abroad, a month before. The violence was such, that it completely broke out from the centre of the shaft five or six inches of the length of the femur, as was found after amputation; and the external wound reached from two inches below the groin to below the knee. Owing to circumstances, amputation could not be performed at the time, and he was sent to England. The thigh was shortened many inches, turned outwards, distorted, and greatly swollen, from the thickened soft parts and large collections of pus. He suffered great pain, was emaciated, and so feeble as not to be able to raise himself in bed. The plan followed by Mr. Fowler, under whose care he was, consisted in giving free exit to the pus by incisions, and in improving his health by generous diet and tonics, until he could bear amputation. During the next two months, under this treatment, he improved much in health and strength, but 